\title{
Pediatric coronary artery fistula: echocardiographic case reports and literature review of treatment strategy
}

\author{
Guang Song ${ }^{1}$, Jing Zhang ${ }^{1}$, Weidong Ren ${ }^{1 *}$, Ying Li ${ }^{1}$ and Ke Zhou $^{2}$
}

Background: Coronary artery fistula (CAF) is a rare cardiac anomaly. Application of transthoracic echocardiography (TTE) is not fully illustrated in pediatric period. Meanwhile, the treatment strategy of CAF is still a controversial issue.

Case presentation: Five cases of CAF with different types were presented. We also retrospectively reviewed 32 records of CAF in our institution from May 2001 to January 2015, including cardiac murmurs, symptoms, TTE diagnoses, complications, other anomalies, treatment and outcome. We summarized the most acceptable treatment strategy for pediatric patients. $71.9 \%$ of all (23/32) had murmurs, $15.6 \%$ of all (5/32) had symptoms. 24 patients received surgery or other imaging examination after TTE. $87.5 \%$ of all (21/24) were correctly and accurately diagnosed by echocardiography, including pointing out the origin and outlet of CAF and complication. During the followup of all 32 patients, there was no spontaneous closure, heart failure, infective endocarditis or death case.

Conclusions: TTE is a useful method that should be considered in the investigation and follow up of pediatric coronary artery fistula. The treatment strategy for pediatric patients should be individuation.

Keywords: Pediatric, Coronary artery fistula, Echocardiography

\section{Background}

Coronary artery fistula (CAF) is a rare cardiac anomaly with incidence of $0.002 \%$ in the general population (Fernandes et al. 1992). CAF comprises $0.31 \%$ of all congenital heart defects (Caldwell and Ensing 1989). Complications secondary to CAF increase with age, including congestive heart failure (CHF), aneurismal dilatation of the coronary artery, infective endocarditis even death. Surgical risk and postoperative complications increase in patients over 20 years of age (Liberthson et al. 1979). So diagnosis as early as possible is good for patients. Transthoracic echocardiography (TTE) has been proved useful in adults and is sufficient for the diagnosis of CAF (Xie et al. 2014). Until now, the treatment strategy of CAF is still a controversial issue (particularly in asymptomatic patients). Herein, we present five different cases of CAF and summarize the acceptable treatment strategy.

\footnotetext{
*Correspondence: renwd01@163.com

1 Department of Ultrasound, Shengjing Hospital of China Medical University, 36\# Sanhao Street, Shenyang 110004, China

Full list of author information is available at the end of the article
}

\section{Case presentation \\ Case 1}

A 14-year-old boy was admitted to our hospital because of being heard cardiac murmur during school physical examination. He didn't have any symptom in normal times. TTE revealed that the right coronary artery (RCA), of which diameter was significantly increased (7.9 mm), coursed to right about $25 \mathrm{~mm}$ (Fig. 1a, arrow). Then an aneurismal dilatation of RCA could be seen with a diameter of $27 \mathrm{~mm}$. In this aneurysm, blood flow like a whirlpool (Fig. 1b, arrow). The blood flow through a fistula ostium, which was on the aneurysm wall, into the right ventricle (Fig. 1c, arrow). The peak velocity of blood flow is $3.5 \mathrm{~m} / \mathrm{s}$ in the diastole (Fig. 1d). The surgery was performed 3 days later. The patient recovered well after surgery.

\section{Case 2}

A 5-year-old girl came to our hospital for medical examination before primary school. She usually had no symptom. Cardiac auscultation could hardly hear any murmur. The electrocardiogram was normal. TTE showed that a 


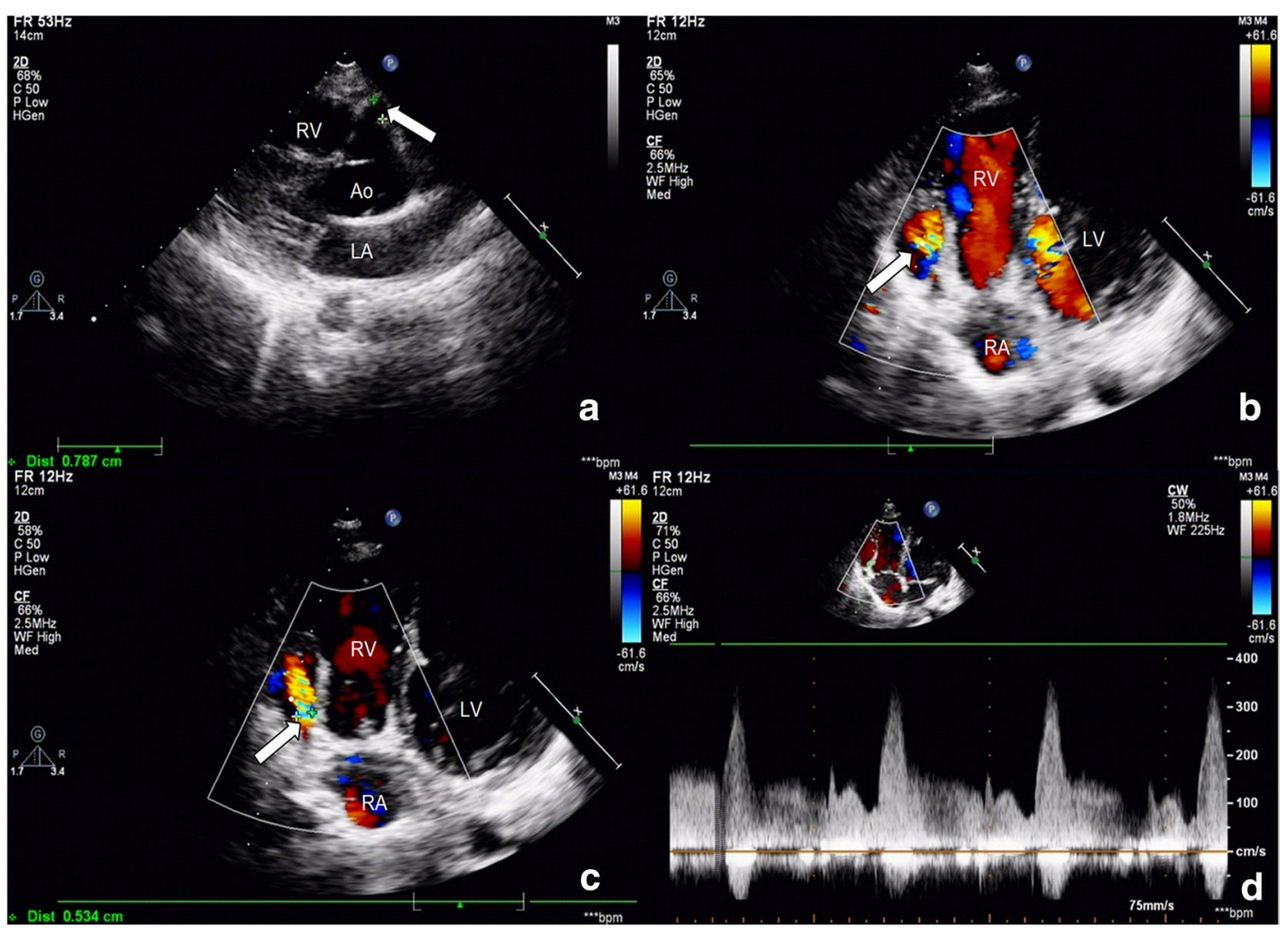

Fig. 1 Right coronary artery-right ventricle (RV) fistula. a Arrow showed the dilated right coronary artery. b Arrow showed the aneurismal dilatation of right coronary artery. c Arrow showed fistula ostium. d The spectrum of fistula was continuous. Ao aorta, $L A$ left atrium, $L V$ left ventricle, $R A$ right atrium, $R V$ right ventricle

diastolic high speed blood flow $(3.0 \mathrm{~m} / \mathrm{s})$ was found on lateral wall of main pulmonary artery above the pulmonary valve annulus $12 \mathrm{~mm}$ (Fig. 2a, arrow). The abnormal blood flow pointed to the medial wall of main pulmonary artery. Reverse tracing of the abnormal blood flow, we found that the source is the left anterior descending (LAD) of left coronary artery (LCA) (Fig. 2b, arrow). The peak of the blood flow was more than $2.0 \mathrm{~m} / \mathrm{s}$ in the diastole (Fig. 2c). After 3 years' follow-up, she was still health.

\section{Case 3}

A 4-year-old girl came to our hospital because of being heard a grade $3 / 6$ continuous murmur at the left side of the chest. She didn't have obvious symptoms. TTE revealed that the diameter of RCA was significantly increased $(9.0 \mathrm{~mm})$ (Fig. 3a, arrow). Track the blood flow, we found that the coronary artery opened at the right atrium and the spectrum of fistula was continuous (Fig. 2b-d). At the same time, mild pulmonary stenosis

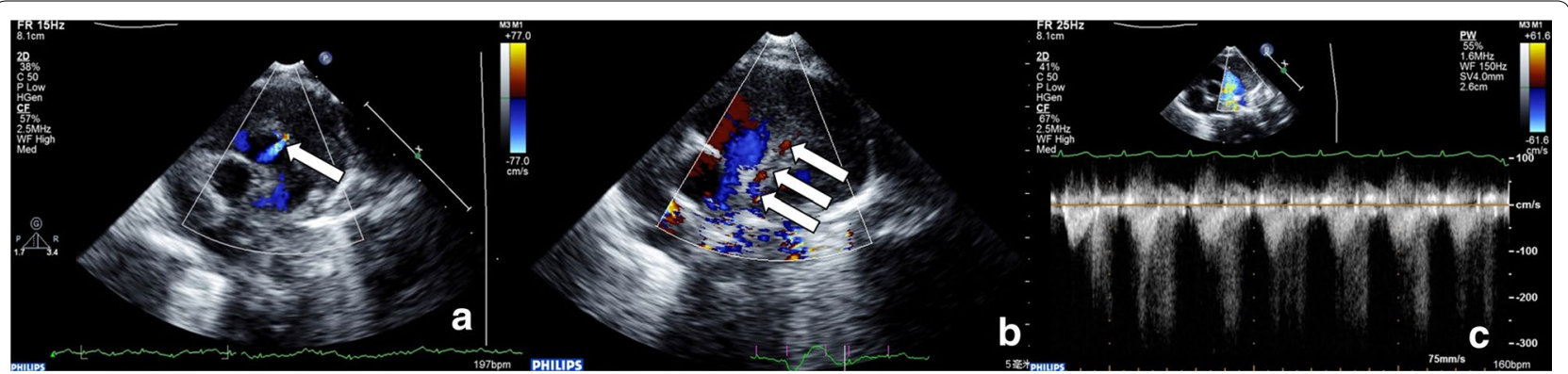

Fig. 2 Left anterior descending-pulmonary artery fistula. a Arrow showed the abnormal blood flow in the pulmonary artery. b Arrow showed the origin of the fistula is left anterior descending. c The spectrum of fistula showed only in diastole 


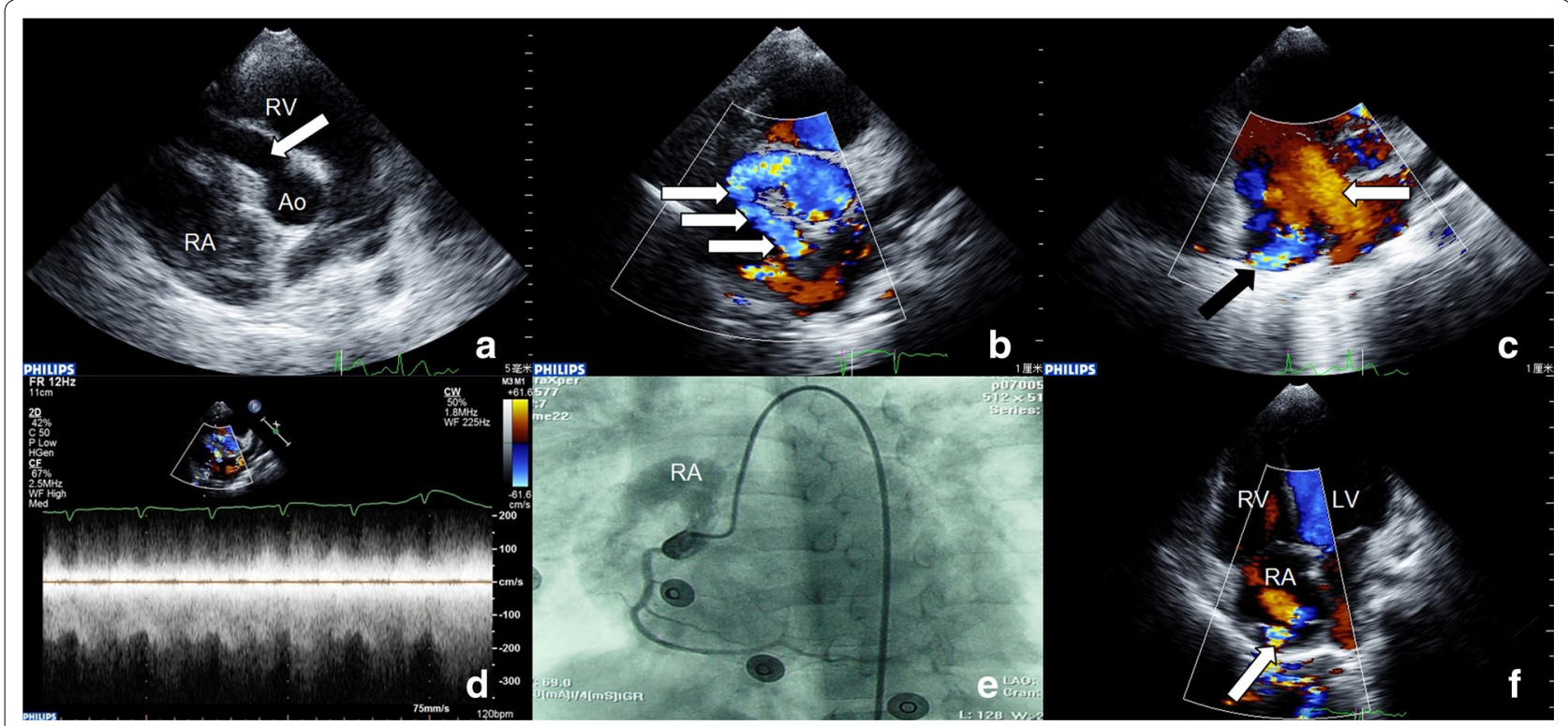

Fig. 3 Right coronary artery-right atrium (RA) fistula. a Arrow showed the dilated right coronary artery. b Arrow showed the course of fistula. c White arrow showed fistula ostium, black arrow showed the atrial septal defect. d The spectrum of fistula is continuous. e Angiography confirmed the diagnosis before surgery. f Arrow showed a tiny residual shunting after surgery. Ao aorta, LV left ventricle, $R A$ right atrium, $R V$ right ventricle

and a big atrial septal defect also have been detected (Fig. 2c, arrow). Angiography confirmed the diagnosis (Fig. 2e). The surgery was performed a week later. One week after surgery, another TTE was performed and found that a residual flow on the top of right atrium (Fig. 2f, arrow). Due to the small shunt volume, the doctor decided to take conservation treatment and followup. The patient was discharged with well recovery.

\section{Case 4}

A 10-year-old girl was admitted to hospital because of a diastolic cardiac murmur of grade $3 / 6 \sim 4 / 6$ in the left side of the chest. TTE showed LAD was dilated $(12 \mathrm{~mm})$ (Fig. 4a, arrow). The LAD coursed to left from the posterior wall of the main pulmonary artery, then around to the front of pulmonary artery. The fistula ostium was on the middle part of ventricular septum (Fig. 4c), and its size was $5 \mathrm{~mm}$. The spectrum of fistula revealed the shunt mainly occurred in diastole and peak speed was more than $4.0 \mathrm{~m} / \mathrm{s}$ (Fig. $4 \mathrm{~d}$ ). Intraoperative findings confirmed the TTE diagnosis (Fig. 4e). One month after surgery, another TTE was performed and found there was a residual flow (Fig. 4f, arrow). The doctor decided not to carry out the second operation. The patient was healthy with follow-up until now.

\section{Case 5}

A 13-day-old male premature infant was admitted to our hospital for medical treatment because of progressive dyspnea. Cardiac auscultation revealed a grade $3 / 6$ continuous murmur at the left side of the chest. TTE showed coronary artery fistula from the left circumflex to coronary sinus (Fig. 5). Computed tomogram angiography confirmed the diagnosis. The doctor decided to take the conservation treatment for the patient. Two weeks later, the patient was discharged with increasingly improving physical condition. The patient is 2.5 years of age now, and is asymptomatic with a normal exercise capacity.

\section{Discussion}

We retrospectively reviewed the medical records of our institution (Department of Ultrasound at the Shenging Hospital of China Medical University) from May 2001 to January 2015 to search for children with CAF (Table 1).

$71.9 \%$ of all (23/32) had murmurs, $15.6 \%$ of all $(5 / 32)$ had symptoms. CAF were diagnosed in 32 patients. $56.3 \%$ of all $(18 / 32)$ had isolated fistula, while the rest 14 patients associated with other cardiac anomalies. Fistula originating from the right coronary artery (RCA) accounted for $59.4 \%$, with $37.5 \%$ from the left coronary artery (LCA), $3.1 \%$ from both coronary arteries. The fistula might drain into the right ventricle (53.1\%), the pulmonary artery $(18.8 \%)$, the right atrium $(18.8 \%)$, the left ventricle $(6.3 \%)$ or the CS (3.1\%). 24 patients received surgery or other imaging examination after TTE, including 19 cases with surgery, 1 case with transcatheter treatment, 4 cases with angiography and 4 cases with 


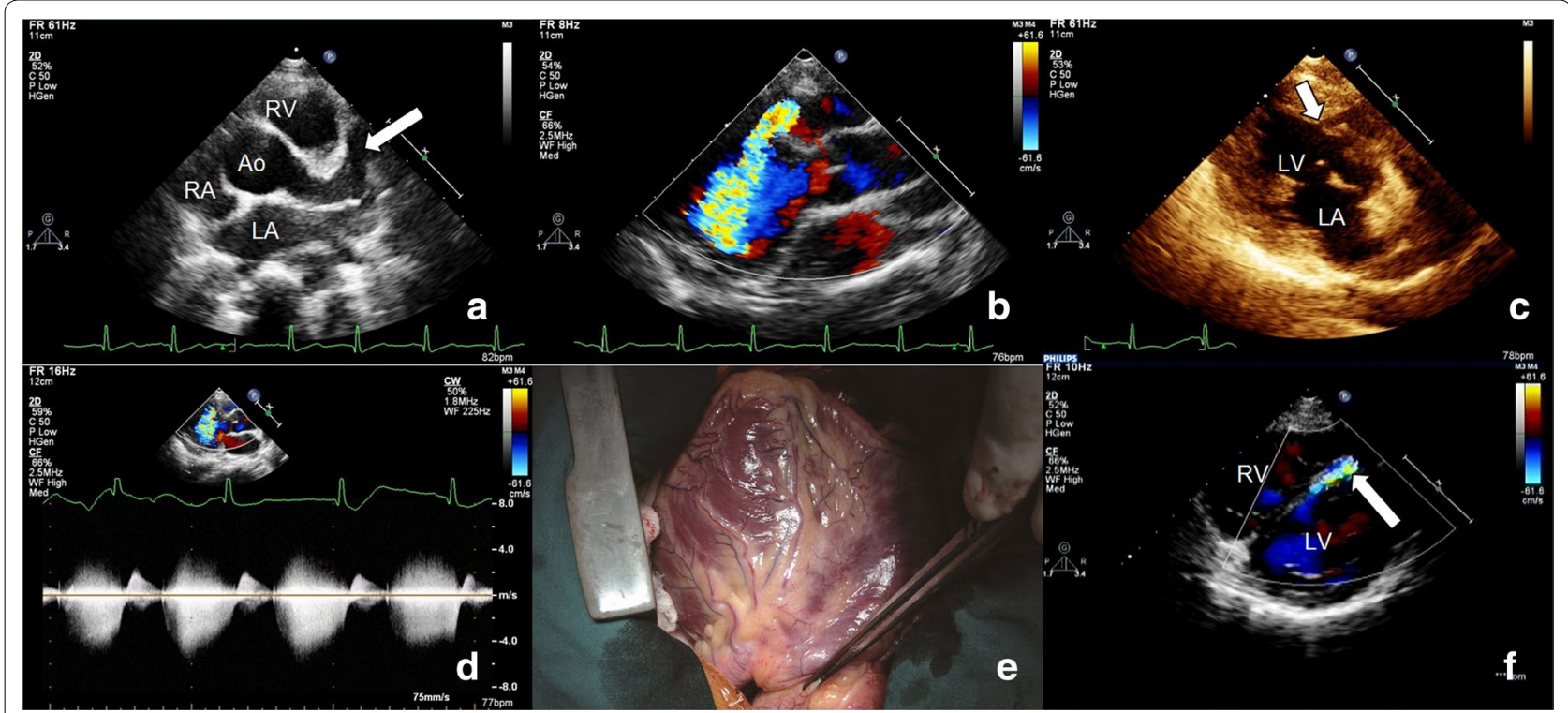

Fig. 4 Left anterior descending-left ventricle (LV) fistula. a Arrow showed the dilated left anterior descending. b Color Doppler showed the course of fistula. c Arrow showed fistula ostium. d The spectrum of fistula showed only in diastole. e Surgery confirmed the diagnosis. f Arrow showed a tiny residual shunting after surgery. Ao aorta, $L A$ left atrium, $L V$ left ventricle, $R A$ right atrium, $R V$ right ventricle

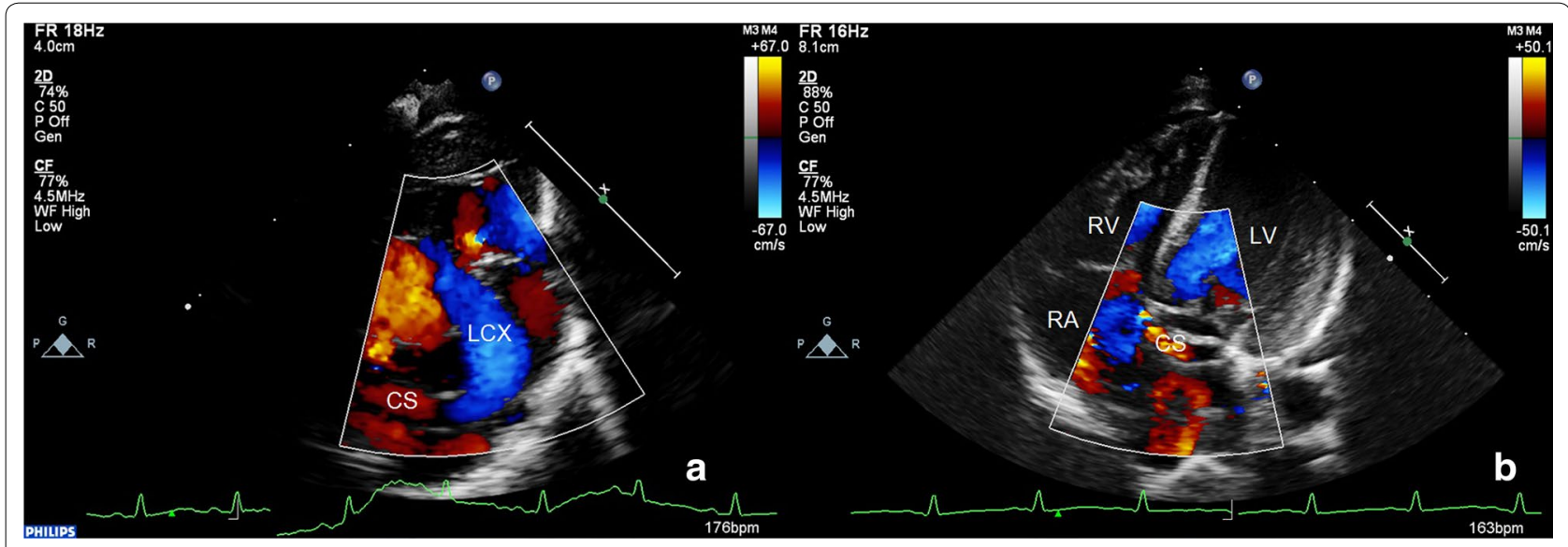

Fig. 5 Left circumflex (LCX)-coronary sinus (CS) fistula. a LCX was dilated and connected with CS. b CS was connected with right atrium (RA). CS coronary sinus, $L C X$ left circumflex, $L V$ left ventricle, $R A$ right atrium, $R V$ right ventricle

computed tomogram angiography. $87.5 \%$ of all $(21 / 24)$ were correctly and accurately diagnosed by echocardiography, including pointing out the origin and outlet of CAF and complication. 3 cases (Nos. 15, 16, 17) could not show the origins of CAF, which are the tiny branches of coronary artery diagnosed by angiography. 12 patients received conservation treatment. During the follow-up of all 32 patients, there was no spontaneous closure, heart failure, infective endocarditis or death case.

The cardiac murmur, the dilated coronary artery and abnormal blood flow could lead the sonographer to suspect the existence of CAF. Although limited with the size of chambers and vessels, CAF was still easily detected by TTE due to pediatric good imaging quality.

The complications of CAF include: (1) aneurysmal dilatation of the coronary artery: aneurysmal dilatation was found in 19-26 \% of CAFs (Said and Gamal 1995; Urrutia et al. 1983). (2) Congestive heart failure: some research revealed that congestive heart failure is reported in $6 \%$ of patients with CAF under age 20 years (Starc et al. 1986). The pulmonary vascular resistance increased in neonatal period and decreased at between 2 and 3 months of age. So congestive heart failure caused by CAF usually presented at between 2 and 3 months of age. Congestive 
Table 1 Clinical data, TTE and outcome

\begin{tabular}{|c|c|c|c|c|c|c|c|c|}
\hline \multirow[t]{2}{*}{ 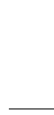 } & \multirow[t]{2}{*}{ Age } & \multirow[t]{2}{*}{ Gender } & \multirow[t]{2}{*}{ Symptoms } & \multicolumn{3}{|l|}{ TTE } & \multirow[t]{2}{*}{ Other imaging } & \multirow[t]{2}{*}{ Treatment } \\
\hline & & & & Origin & Outlet & Other & & \\
\hline 1 & $5 Y$ & M & Murmur & RCA & $\mathrm{RV}$ & None & & Surgery \\
\hline 2 & $2 Y$ & $F$ & Murmur & RCA & $\mathrm{RV}$ & None & CTA & Surgery \\
\hline 3 & $7 Y$ & M & Fatigue & RCA & $\mathrm{RV}$ & Aneurysmal dilatation & & Surgery \\
\hline 4 & $14 Y$ & M & Murmur & RCA & $\mathrm{RV}$ & Aneurysmal dilatation & & Surgery \\
\hline 5 & $1 Y$ & $\mathrm{~F}$ & Murmur & LAD & $\mathrm{RV}$ & None & & Surgery \\
\hline 6 & $3 Y$ & M & Murmur & RCA & $\mathrm{RV}$ & None & & Surgery \\
\hline 7 & $16 Y$ & M & None & $L A D$ & RV & None & & Surgery \\
\hline 8 & $2 Y$ & $\mathrm{~F}$ & Murmur & RCA & $\mathrm{RV}$ & PS & & Surgery \\
\hline 9 & $2 Y$ & M & None & RCA & $\mathrm{RV}$ & None & & Surgery \\
\hline 10 & $8 Y$ & $F$ & Murmur & RCA & $\mathrm{RV}$ & None & & Surgery \\
\hline 11 & $5 Y$ & $F$ & Murmur & $\mathrm{RCA}$ & RV & VSD & & Surgery \\
\hline 12 & $10 Y$ & $\mathrm{~F}$ & Murmur & RCA & RV & None & & Transcatheter closure \\
\hline 13 & $52 d$ & $\mathrm{~F}$ & Murmur & $\mathrm{RCA}$ & $\mathrm{RV}$ & $\mathrm{PS}, \mathrm{CHF}$ & & Conservation \\
\hline 14 & $44 d$ & $\mathrm{~F}$ & Murmur & RCA & RV & None & & Conservation \\
\hline 15 & $60 d$ & M & None & LCA & RV & None & & Conservation \\
\hline 16 & $1 d$ & M & None & RCA & RV & None & & Conservation \\
\hline 17 & $2 d$ & $F$ & Murmur dyspnea & LCA & RV & PAH & & Conservation \\
\hline 18 & $5 Y$ & $\mathrm{~F}$ & Murmur & RCA LAD & PA & Mitral valve prolapse & & Surgery \\
\hline 19 & $11 Y$ & $F$ & None & Unknown & PA & None & RCA by angiography & Conservation \\
\hline 20 & $5 Y$ & M & None & Unknown & PA & None & RCA by angiography & Conservation \\
\hline 21 & $6 Y$ & M & Dyspnea & Unknown & PA & None & LCX by angiography & Conservation \\
\hline 22 & $6 Y$ & M & Murmur & LAD & PA & ASD & & Conservation \\
\hline 23 & $5 \mathrm{M}$ & $\mathrm{F}$ & None & LAD & PA & None & & Conservation \\
\hline 24 & $4 Y$ & $F$ & Murmur & LCA & RA & None & CTA & Surgery \\
\hline 25 & $1 Y$ & $F$ & Murmur & RCA & RA & $\mathrm{CHF}, \mathrm{PAH}$ & & Surgery \\
\hline 26 & $7 Y$ & $F$ & Murmur & $\mathrm{RCA}$ & RA & PE & & Surgery \\
\hline 27 & $4 Y$ & $F$ & Murmur & $\mathrm{RCA}$ & RA & ASD,PS & Angiography & Surgery \\
\hline 28 & $7 Y$ & $F$ & Murmur & LCA & RA & None & & Surgery \\
\hline 29 & $57 d$ & $\mathrm{~F}$ & Murmur & RCA & RA & PFO & & Conservation \\
\hline 30 & $11 Y$ & $\mathrm{~F}$ & Murmur & LAD & LV & None & CTA & Surgery \\
\hline 31 & $1 Y$ & $F$ & Dyspnea murmur & LCX & LV & VSD, PDA, PAH & & Surgery \\
\hline 32 & $13 d$ & M & Dyspnea murmur & LCX & CS & CS stenosis & CTA & Conservation \\
\hline
\end{tabular}

$R C A$ right coronary artery, $L C A$ left coronary artery, $L C X$ left circumflex, $L A D$ left anterior descending, $R V$ right ventricle, $P A$ pulmonary artery, $R A$ right atrium, $L V$ left ventricle, $C S$ coronary sinus, $P S$ pulmonary stenosis, $C H F$ congestive heart failure, $A S D$ atrial septal defect, $P A H$ pulmonary arterial hypertension, $P F O$ patent foramen ovale, $P E$ pericardial effusion, VSD ventricular septal defect, PDA patent ductus arteriosus

heart failure in neonatal period is rare. The termination of the fistula into low-pressure cardiac structures such as the right atrium and coronary sinus is also likely to result in a larger left-to-right shunting than if the fistula terminated in high-pressure structures like the left ventricle (Li et al. 2011). (3) infective endocarditis: the incidence of infective endocarditis with patients under 20 years old is $3 \%$. Meanwhile, the fistula usually was medium or large (Liberthson et al. 1979). Due to the very early diagnosis, all patients in our study got the diagnosis before they have serious symptoms or complications. So the very early diagnosis is good for patients, especially for those over 20 years of age (Liberthson et al. 1979).

Current treatment options include conservation treatment, surgery and transcatheter closure. The management of CAF is still a controversial issue (particularly in asymptomatic patients).

Treatment strategy for asymptomatic CAF: (1) small/ low-flow shunting fistula do not require a specific procedure for closure simply (like our case 2). However, closure of fistula may be recommended: (1) if the patient is considered to be at high risk for later infective endocarditis; 
(2) if longitudinal follow-up is not feasible; (3) if the patient is undergoing an invasive procedure for some other cardiac problem (Latson 2007). (2) Big/high-flow shunting: patients need surgery or transcatheter closure although fistula may not produce symptoms during infancy or childhood. There is a risk that heart failure may develop owing to chronic left ventricular volume overload later in life (Mavroudis et al. 1997). Also, the incidence of death increase significantly after two decades (Hong et al. 2004).

Treatment strategy for symptomatic CAF: (1) neonate/ infant: symptomatic CAFs have been associated with significant morbidity and mortality. Surgery can be performed with comparatively low risk and is essentially curative. The symptoms from a large left-to-right shunting are often controllable by typical medical management. Eventually $50 \%$ of fistulas become asymptomatic due to decrease in relative size of the fistula and shunting with growth, or spontaneous regression (Hsieh et al. 2002). Those who do not respond to medical management need intervention. (2) elder: elective closure of CAF either by surgery or by transcatheter techniques should be taken into consideration.

The first successful surgical was operated by Bjork and Crafoord in 1947, and the first therapeutic embolization was performed by Zuberbuhler et al. in 1974. Previous study showed that a very low morbidity and mortality rate $(0-4 \%)$ when fistulas are operated on in infancy and childhood (Schumacher et al. 1997). Although repair of coronary artery fistula is relatively mature, postoperative complications may happen occasionally, including thrombosis, angina with coronary thrombosis, myocardial infarction and rupture of aneurysm. The incidence of each is less than $2 \%$ (Mangukia 2012). There are also $20-30 \%$ of patients with residual shunting using coronary angiography after CAF repair. Meanwhile, $10 \%$ had demonstrable recurrence of fistula without hemodynamic disturbance (Cheung et al. 2001; Valente et al. 2010). Although postoperative coronary angiography should be done, some parents refused to perform it because of invasion and radiation. So we use TTE to evaluate the effect of surgery and to follow up just like Case 3.

In conclusion, TTE is a useful method that should be considered in the investigation and follow up of pediatric coronary artery fistula. The treatment strategy for pediatric patients should be individuation.

\section{Consent}

This study was approved by the Regional Ethics Committee of our hospital and that permission was granted to all the authors to access the patient's data. Written informed consents were obtained from legal parents of all patients for the publication of this report and any accompanying images.
Abbreviations

CAF: coronary artery fistula; TTE: transthoracic echocardiography.

\section{Authors' contributions}

GS and WR designed the study; GS drafted the manuscript. JZ, YL and KZ collected the cases. All authors read and approved the final manuscript.

\section{Author details}

${ }^{1}$ Department of Ultrasound, Shengjing Hospital of China Medical University, 36\# Sanhao Street, Shenyang 110004, China. ${ }^{2}$ Department of Cardiac Surgery, Shengjing Hospital of China Medical University, Shenyang, China.

\section{Acknowledgements}

None.

\section{Competing interests}

The authors declare that they have no competing interests.

Received: 18 February 2016 Accepted: 8 September 2016

Published online: 15 September 2016

\section{References}

Caldwell RL, Ensing GJ (1989) Coronary artery abnormalities in children. J Am Soc Echocardiogr 2:259-268

Cheung DL, Au WK, Cheung HH, Chiu CS, Lee WT (2001) Coronary artery fistulas: long-term results of surgical correction. Ann Thorac Surg 71:190-195

Fernandes ED, Kadivar H, Hallman GL, Reul GJ, Ott DA, Cooley DA (1992) Congenital malformations of the coronary arteries: the Texas Heart Institute experience. Ann Thorac Surg 54:732-740

Hong GJ, Lin CY, Lee CY et al (2004) Congenital coronary artery fistulas: clinical considerations and surgical treatment. ANZ J Surg 74:350-355

Hsieh KS, Huang TC, Lee CL (2002) Coronary artery fistulas in neonates, infants, and children: clinical findings and outcome. Pediatr Cardiol 23:415-419

Latson LA (2007) Coronary artery fistulas: how to manage them. Catheter Cardiovasc Interv 70:110-116

Li J, Sakazaki H, Tsukuda K, Fujiwara K (2011) A neonate of coronary artery fistula from left circumflex to coronary sinus. Pediatr Cardiol 32:1212-1215

Liberthson RR, Sagar K, Berkoben JP, Weintraub RM, Levine FH (1979) Congenital coronary arteriovenous fistula. Report of 13 patients, review of the literature and delineation of management. Circulation 59:849-854

Mangukia CV (2012) Coronary artery fistula. Ann Thorac Surg 93:2084-2092

Mavroudis C, Backer CL, Rocchini AP, Muster AJ, Gevitz M (1997) Coronary artery fistulas in infants and children: a surgical review and discussion of coil embolization. Ann Thorac Surg 63:506-512

Said SA, el Gamal MI (1995) Coronary angiographic morphology of congenital coronary arteriovenous fistulas in adults: report of four new cases and review of angiograms of fifteen reported cases. Cathet Cardiovasc Diagn 35:29-35

Schumacher G, Roithmaier A, Lorenz HP et al (1997) Congenital coronary artery fistula in infancy and childhood: diagnostic and therapeutic aspects. Thorac Cardiovasc Surg 45:287-294

Starc TJ, Bowman FO, Hordof AJ (1986) Congestive heart failure in a newborn secondary to coronary artery-left ventricular fistula. Am J Cardiol 58:366-367

Urrutia-S CO, Falaschi G, Ott DA, Cooley DA (1983) Surgical management of 56 patients with congenital coronary arteriovenous fistulas. Ann Thorac Surg 35:300-307

Valente AM, Lock JE, Gauvreau K et al (2010) Predictors of long-term adverse outcomes in patients with congenital coronary artery fistulae. Circ Cardiovasc Interv 3:134-139

Xie M, Li L, Cheng TO et al (2014) Coronary artery fistula: comparison of diagnostic accuracy by echocardiography versus coronary arteriography and surgery in 63 patients studied between 2002 and 2012 in a single medical center in China. Int J Cardiol 176:470-477 